\title{
Mixed Stack Charge Transfer Crystals: Crossing the Neutral-Ionic Borderline by Chemical Substitution
}

\author{
Nicola Castagnetti, Matteo Masino, Corrado Rizzoli, and Alberto Girlando \\ Dipartimento di Scienze Chimiche, della Vita e della Sostenibilità Ambientale (SCVSA) and INSTM-UdR Parma, \\ Università di Parma, Parco Area delle Scienze, IT-43124 Parma, Italy \\ Concepció Rovira \\ Department of Molecular Nanoscience and Organic Materials, \\ Institut de Ciència de Materials de Barcelona (ICMAB-CSIC) and Networking Research Center on Bioengineering, \\ Biomaterials and Nanomedicine (CIBER-BBN), ES-08193 Bellaterra, Spain
}

(Dated: January 19, 2018)

\begin{abstract}
We report extensive structural and spectroscopic characterization of four mixed stack chargetransfer (ms-CT) crystals formed by the electron donor 3,3',5,5'-tetramethylbenzidine (TMB) with Chloranil (CA), Bromanil (BA), 2,5-difluoro-tetracyanoquinodimethane ( $\mathrm{TCNF}_{2}$ ) and tetrafluorotetracyanoquinodimethane $\left(\mathrm{TCNQF}_{4}\right)$. Together with the separately studied TMB-TCNQ [Phys. Rev. B 95, 024101 (2017)] the TMB-acceptor series span a wide range of degree of CT, from about 0.14 to 0.91 , crossing the neutral-ionic interface, yet retaining similar packing and donor-acceptor CT integrals. First principle calculations of key phenomenological parameters allows us to get insight into the factors determining the degree of CT and other relevant physical properties.
\end{abstract}

\section{INTRODUCTION}

Charge transfer (CT) crystals constitute a wide family of molecular solids that include organic semiconductors, metals, superconductors, and ferroelectric materials [1-3]. Interest has recently flowered around a particular class of systems, the so-called mixed stack (ms) CT crystals, where $\pi$-electron donor (D) and acceptor (A) molecules alternate to form one-dimensional ..DADADA $\cdots$ chains $[4,5]$. The overlap between frontier molecular orbitals along the stack leads to delocalized electrons in one dimension, and the D/A character of molecular units leads to alternating on-site charge $\cdots \mathrm{D}^{\rho+} \mathrm{A}^{\rho-} \mathrm{D}^{\rho+} \mathrm{A}^{\rho-} \ldots$ along the stack.

In ms-CT crystals the specific value of $\rho$, the degree of charge transfer or ionicity, depends on the relative electron-donating/accepting strength of the two molecules and on the intermolecular hopping $t$, but is also affected by electrostatic intermolecular interactions. The importance of long range electrostatic interaction (Madelung energy) emerged very clearly with the discovery of the neutral-ionic phase transition (NIT) $[6,7]$. In this peculiar phase transition a ms-CT crystal passes from a formally neutral $(\rho \lesssim 0.5)$ to a formally ionic $(\rho \gtrsim 0.5)$ ground state as a consequence of the increase of Madelung energy following the lattice compression induced by temperature or pressure. Furthermore, on the ionic side the stack is unstable due the Peierls mechanism, so that NIT is always accompanied by stack dimerization [8]. The interplay between correlated electrons and both inter- and intra-molecular phonons enhances the complexity of the system both in terms of the appearance of new phases and of amplification of materials responses. Mixed stack CT crystals are then characterized by an intriguing phenomenology that includes multiple competing phases, divergent response, and anomalous metallic states [9]. From the perspective of materials research, several application oriented aspects are being pursued, that span from organic semiconductivity and photovoltaic $[10,11]$, to ultrafast photoswitching [12], to ferroelectrics [13], to cite a few.

Since the above described multivariate phenomenology depends on the interplay of the different interactions involved, it is important to explore the phase space encompassed by different ms-CT crystals to understand and ultimately control the factors determining the physical properties of interest. Such a task has recently started in the field of organic semiconductors, with the aim of engineering systems with different band gaps and degree of CT, in such a way to understand how these parameters affect the semiconducting behavior [14-16]. However, this systematic exploration has been so far limited to ms-CT crystals in the proximity of the neutral side $(0 \lesssim \rho \lesssim 0.2)$, as a rather weak electron donor has been associated with acceptors of increasing electron affinity. Here we couple a relatively strong electron donor, 3,3',5,5'-tetramethylbenzidine (TMB) with an extensive series of different electron acceptors, in such a way to span the ionicity from the neutral to the ionic side. Evaluation of the most important phenomenological parameters entering the modified Hubbard model apt to describe and understand a large spectrum of experimental data [17] allows us to get insight into the factors determining the crystal packing and physical properties of the systems under investigation.

\section{EXPERIMENTAL AND COMPUTATIONAL METHODS}

\section{A. Crystal growth}

Two different methods have been used for the crystal growth: Slow solvent evaporation of a saturated solu- 
tion of the donor and acceptor molecules in (1:1) ratio, and sublimation in an open tube under controlled atmosphere (physical vapor transport method, PVT) [18]. Good quality crystals of TMB with chloranil (CA) and bromanil (BA) have been obtained by the first method, starting from acetonitile solution. Crystals of TMB with 2,5-difluoro- and tetrafluoro-tetracyanoquinodimethane $\left(\mathrm{TCNQF}_{2}\right.$ and $\left.\mathrm{TCNQF}_{4}\right)$ could be obtained only by the second method. Neither method provided good quality crystals of TMB-FA (FA: fluoranil).

\section{B. X-ray analysis}

Single crystals were mounted on a Bruker SMART 1000, Bruker APEXII or Bruker DUO VENTURE diffractometer equipped with graphite monochromated Mo $\mathrm{K} \alpha$ radiation $(\lambda=0.71073 \AA)$ fine-focus sealed tube. The intensity data were collected using $\omega$ scan at 294(2) $\mathrm{K}$ and $150 \mathrm{~K}$ in the case of $\mathrm{TMB}^{-\mathrm{TCNQF}_{4}}$. Cell refinements and data reductions were performed using the Bruker SAINT software [19]. The structures were solved by direct methods using the program SHELXT [20], and refined with full-matrix least-squares based on $F^{2}$ using the program SHELXL2014/7 [21]. In TMB-TCNQF 2 the fluorine atoms are disordered over two sets of sites with refined occupancy ratio 0.691(4):0.309(4). All nonhydrogen atoms were refined anisotropically, while in TMB-CA they were located in a difference Fourier map and refined freely. The N-bound hydrogen atoms were located in a difference Fourier map (TMB-TCNQF 4 ) or placed geometrically $\left(\mathrm{TMB}-\mathrm{TCNQF}_{2}\right)$ and refined freely $\left(\mathrm{TMB}_{-} \mathrm{TCNQF}_{4}\right)$ or with $U_{i s o}(\mathrm{H})=1.2 U_{e q}(\mathrm{~N})$. The N$\mathrm{H}$ bond length in TMB-TCNQF 2 was constrained to be 0.86(1) A. Except for TMB-CA, all C-bound hydrogen atoms were placed geometrically and refined using a riding model approximation, with $\mathrm{C}-\mathrm{H}=0.93-0.97 \AA$ and with $U_{i s o}(\mathrm{H})=1.2 U_{e q}(\mathrm{C})$ or $1.5 U_{e q}(\mathrm{C})$ for methyl $\mathrm{H}$ atoms. The molecular graphics were prepared using Mercury 3.9 [22] and Jmol [23] programs.

\section{Spectroscopic measurements}

Infrared (IR) spectra of the crystals were recorded with a Bruker IFS 66 Fourier transform IR (FT-IR) spectrometer coupled to an IR microscope Hyperion 1000. Spectral resolution: $2 \mathrm{~cm}^{-1}$. We used a wire-grid polarizer and a Polaroid to polarize the light in the mid-IR and near-IR regions, respectively, and a gold mirror as reference in the reflectance measurements. Due to surface irregularities of the samples, the reflectance values cannot be considered as absolute. The Raman spectra were recorded with a Renishaw 1000 Raman spectrometer equipped with the appropriate edge filter, and coupled to a Leica M microscope. A Lexel Kr laser was used as light source. Exciting lines: $676.4 \mathrm{~nm}$ for TMB-CA and TMB-BA, $647.2 \mathrm{~nm}$ for $\mathrm{TCNQF}_{\mathrm{x}}$.

\section{Computational methods}

We have in part followed the approach recently developed by some of us [24]. The method uses groundstate Density Functional (DFT) calculations as implemented in Gausssian09 [25] for individual molecules and DA dimers, and on the atomistic modeling of intermolecular electrostatic interactions. All calculations have been performed in vacuum using the unrestricted $\omega$ B97XD hybrid functional together with the $6-31+\mathrm{G}^{*}$ basis set. The average charge residing on the $\mathrm{D}$ and $\mathrm{A}$ molecules is estimated on the basis of the computed Hirshfeld atomic charges. The electrostatic interaction $V$ within a DA pair and the Madelung energy $M$ are computed by adopting the point-charge approximation of the molecular charge density based on ESP atomic charges computed for neutral/charged molecules and lattices. Crystal electrostatic sums have been obtained for finite clusters of increasing size using the MESCAL code [26].

\section{RESULTS}

\section{A. TMB-haloquinones}

We have started by coupling TMB with haloquinones of increasing electron affinity, namely tetrafluoro-, tetrachloro- and tetrabromo- $p$-benzoquinone (FA, CA and BA, respectively). We had problems with the TMBFA: Despite several attempts, also by different methods (see Section II A), the crystals, when obtained, often contained impurities and/or where not stable, probably due to spontaneous sublimation of FA. We were able to single out a small crystal for X-ray analysis, but we could only get the structural class [27] and the cell parameters, as reported in Table I. From the frequency of the

TABLE I. Basic structural parameters of TMB-haloquinoes

\begin{tabular}{lccc}
\hline \hline & & & \\
& & & \\
& & & \\
Class & $C 2 / m, Z=2(i)$ & $C 2 / m, Z=2(i)$ & $C 2 / m, Z=2(i)$ \\
$a(\AA)$ & $7.745(3)$ & $20.507(10)$ & $20.492(5)$ \\
$b(\AA)$ & $14.511(6)$ & $6.444(3)$ & $6.5073(15)$ \\
$c(\AA)$ & $20.492(5)$ & $8.790(5)$ & $9.063(2)$ \\
$\beta(\mathrm{deg})$ & $97.526(7)$ & $111.144(9)$ & $110.232(3)$ \\
$V_{\text {cell }}\left(\AA^{3}\right)$ & 984.837 & 1083.37 & 1133.96 \\
\hline \hline
\end{tabular}

antisymmetric $\mathrm{C}=\mathrm{O}$ stretching $\left(1670 \mathrm{~cm}^{-1}\right.$ in the IR absorption spectrum) we deduce $\rho \simeq 0.18$, by assuming for FA a ionization frequency shift of $160 \mathrm{~cm}^{-1}$ analogous to that of CA and BA [28, 29]. However, the vibrational spectra also showed small but significant differences even between crystals of the same batch, indicating the probable presence of polymorphism and/or impurity contamination. For these reason we shall not discuss TMB-FA any further. 


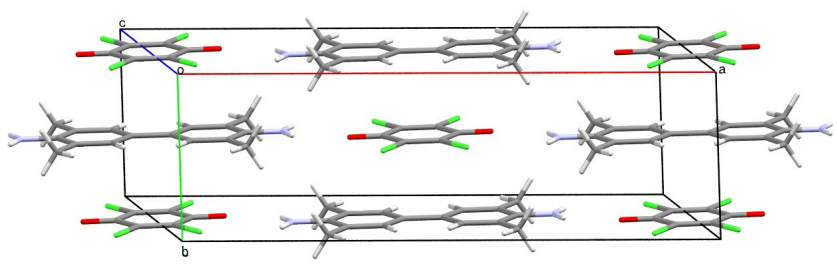

FIG. 1. Crystal structure of the isomorphous TMB-CA and TMB-BA. Shown structure: TMB-CA.

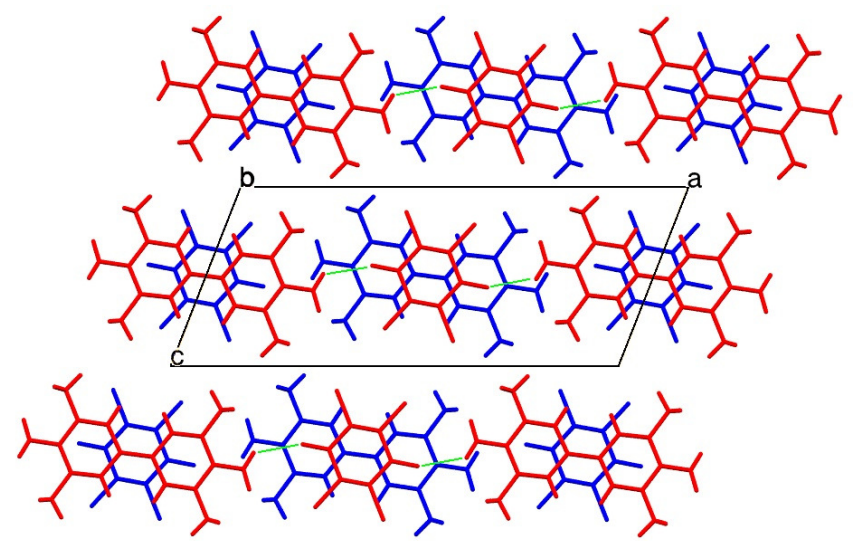

FIG. 2. Crystal structure of TMB-CA (and the isomorphous TMB-BA) viewed from the $b$ stack axis. Red and blue color distinguish molecules lying on different planes parallel to $a c$. The green lines put in evidence the $\mathrm{O} \cdots \mathrm{HN}$ contacts

TMB-CA and TMB-BA are isomorphous. They crystallize in the same class of TMB-FA (space group $C 2 / m, Z=2$ ), but have a different packing, as clearly seen from Table I. The complete crystallographic cif files are reported as Supplemental Material. The TMB-CA packing is reported in Fig. 1. The molecules alternate along the $b$ axis, with the molecular planes perpendicular to it, so that their distance is a half the axis length. Each molecule resides on inversion center, hence the interplanar distance is constant (regular stack). The TMB-BA interplanar distance is slightly higher than that of TMBCA (3.25 vs. $3.22 \AA$ ) due to the bulkier Br atoms. Fig. 2 shows the packing perpendicular to the $b$ stack axis. The red and blue color distinguishes molecules lying on different planes. It is seen that the long axes of the molecules are aligned with each other, almost parallel to the $a$ crystal axis. Furthermore, TMB and CA (BA) molecules alternate along $a$, connected by a network of $\mathrm{O} \cdots \mathrm{HN}$ contacts, while molecules of the same species are found along $c$. The $\mathrm{O} \cdots \mathrm{HN}$ contacts are probably at the origin of the planarity of the $\mathrm{NH}_{2}$ groups of TMB.

Since TMB-CA and TMB-BA are isomorphous, it is convenient to discuss the vibrational spectra in parallel. In this paper we shall not deal with the details of the vibrational assignment, that is any case can be easily obtained on the basis of the available interpretation of the spectra of the neutral molecules and their ions $[29,30]$.

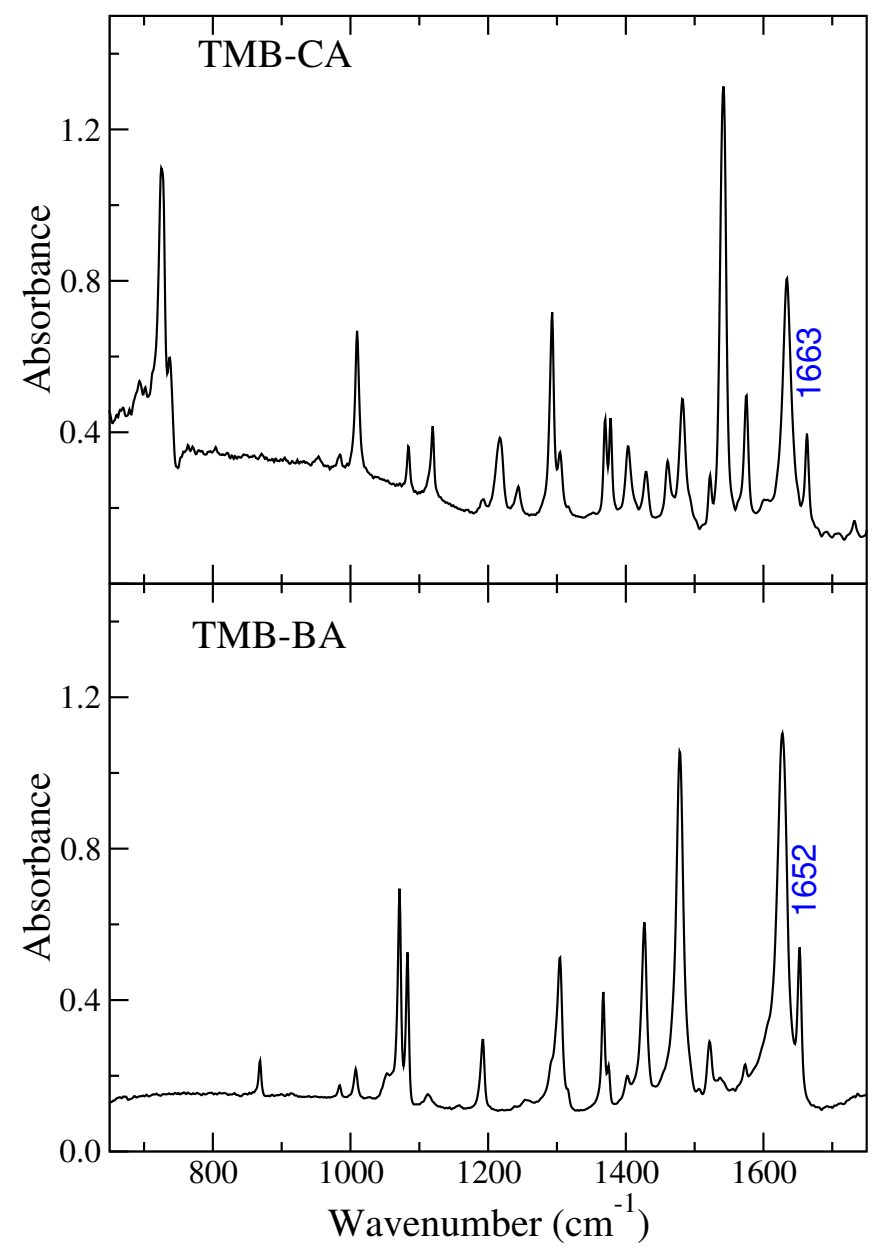

FIG. 3. IR spectra of TMB-CA (top) and TMB-BA (bottom) polarized perpendicularly to the stack axis.

We shall instead limit ourselves to the identification of the charge sensitive, in-plane modes [29, 31], that show up in the infrared (IR) spectra polarized perpendicular to the stack, and to the modes indicative of the stack dimerization, occurring in the IR spectra polarized along the stack, at the same frequency of the Raman spectra $[8,32]$.

Fig. 3 shows the IR absorption spectra of TMB-CA and TMB-BA, collected with electric vector polarized perpendicular to the stack axis. According the above described crystal structure, the charge-sensitive modes will show up only in these spectra. In the present case, we make reference to the only $\mathrm{C}=\mathrm{O}$ antisymmetric stretching mode, because it is easily identified and has a quite large $\left(\sim 160 \mathrm{~cm}^{-1}\right)$ ionization frequency shift [29]. The frequencies of this mode are reported directly in Fig. 3, and yield $\rho=0.14$ and 0.16 for TMB-CA and TMB-BA, respectively. These values are somewhat smaller than the corresponding ones of TTF-CA [33], the prototype CT crystal undergoing NIT [6, 7].

Fig. 4 reports the TMB-CA and TMB-BA IR spectra polarized along the $b$ stack axis. As expected, these 


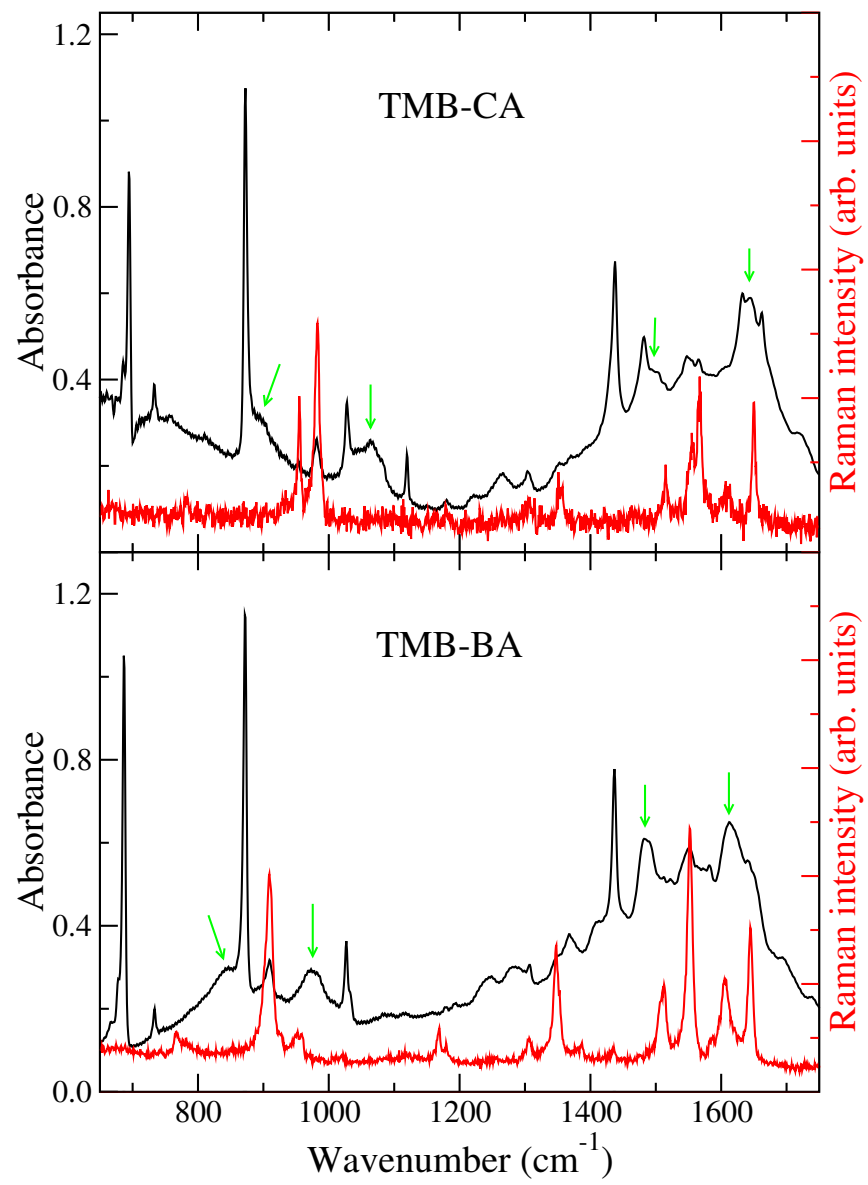

FIG. 4. IR spectra of TMB-CA (top) and TMB-BA (bottom) polarized parallel to the stack axis, compared to the corresponding unpolarized Raman spectra (red line).

spectra are dominated by out-of-plane vibrations. In the Figure we also show, superimposed to the IR, the unpolarized Raman spectra (red trace), in order to verify the occurrence of the so-called sidebands, signatures of the presence of a soft mode [33]. These are somewhat difficult to identify, particularly in TMB-CA due to the presence of overlapping bands. However, a pair of sidebands per spectrum can be singled out, as indicated by the green arrows in the Figure. The frequency difference between the sidebands and the corresponding Raman central peak is about 80 and $65 \mathrm{~cm}^{-1}$ for TMB-CA and TMB-BA, respectively. These frequencies are interpreted as the frequency of the effective soft mode [33, 34] which ultimately might yield to the Peierls distortion [8]. However, we did not observe sign of phase transitions down to $77 \mathrm{~K}$.

In correspondence with the Raman peaks connected to sidebands, and assigned to totally-symmetric modes, we observe a very weak IR band located at the same frequency. This frequency coincidence cannot be accidental, in particular for the rather well isolated bands at 981 and $910 \mathrm{~cm}^{-1}$, assigned to the $a_{g} \nu_{3}$ mode of CA and BA, respectively [29], lowered by the electron-molecular
TABLE II. Basic structural parameters of TMB-TCNQF ${ }_{x}$

\begin{tabular}{l|ccc}
\hline \hline & & & \\
& TMB-TCNQ $^{a}$ & TMB-TCNQF $_{2}$ & TMB-TCNQF $_{4}$ \\
\hline Class & $P 2_{1} / n, Z=2(i)$ & $P 2_{1}, Z=2(1)$ & $C 2 / m, Z=2(i)$ \\
$a(\AA)$ & $6.708(3)$ & $6.7029(11)$ & $8.831(6)$ \\
$b(\AA)$ & $21.797(7)$ & $21.878(4)$ & $22.852(16)$ \\
$c(\AA)$ & $8.074(3)$ & $8.1973(9)$ & $6.679(4)$ \\
$\beta($ deg $)$ & $100.35(5)$ & $100.595(9)$ & $116.499(9)$ \\
$V_{\text {cell }}\left(\AA^{3}\right)$ & 1161.33 & 1181.61 & 1206.26 \\
\hline \hline
\end{tabular}

${ }^{a}$ Ref. [30]

vibration (e-mv) interaction [32]. The frequency coincidence means that the same mode is active in Raman and IR, in contradiction with the mutual exclusion rule implied by the X-ray, as both TMB and haloquinones reside on inversion center. In other words, the vibrational spectra signal that the stack is slightly dimerized, and not regular as indicated by X-ray. Since the IR bands are very weak, the distortion is indeed very small, and might be due to static or dynamic disorder induced by the TMB methyl group. In fact, X-ray detect long range order only, whereas Raman-IR coincidence is indicative of a local distortion. Furthermore, the position of hydrogens is not precisely identified by X-ray, and some disorder in the methyl groups is certainly present in the case of TBM-BA (cf. the corresponding cif file in the Supplemental Material).

\section{B. TMB-TCNQF ${ }_{x}$}

We have recently reported a detailed characterization of the ms-CT crystal made by TMB with TCNQ [30]. At room temperature, TMB-TCNQ crystallizes in the monoclinic system, space group $P 2_{1} / n, Z=2$, and the degree of $\mathrm{CT}$ is $\rho=0.29$. Around $200 \mathrm{~K}$, TMB-TCNQ undergoes a valence instability, with a slight increase of $\rho$ to 0.41 . In this paper we are not dealing with phase transitions, focusing on ambient temperature characterization.

Since TMB-TCNQ undergoes a valence instability at relatively high temperature, we decided to couple TMB with two stronger acceptors, $\mathrm{TCNQF}_{2}$ and $\mathrm{TCNQF}_{4}$. The X-ray crystal structure data are compared in Table II with those of TMB-TCNQ.

The crystal structure of TMB-TCNQF 2 looks like it were derived from that of TMB-TCNQ: The stacking axis is the same $(a)$ and the cell dimensions are similar (Table II). However, there is a loss of the inversion center and of the plane of symmetry. Loss of the inversion center means that the stacks are dimerized, as clearly shown in Fig. 5. However, at variance with the low-temperature phase of TTF-CA [35], the arrangement of the two DA pairs in the unit cell is anti-ferroelectric. The molecules deviate from planarity, possibly due to $\mathrm{CN} \cdots \mathrm{H}_{2} \mathrm{~N}$ contacts. As 


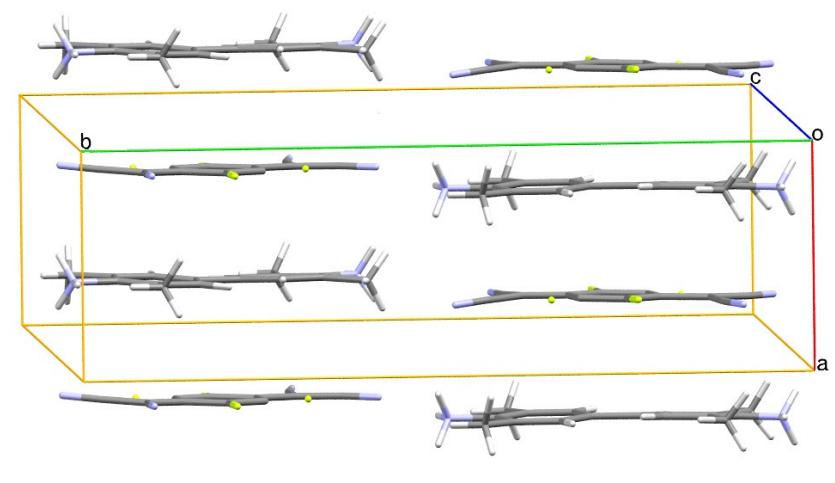

FIG. 5. Crystal structure of TMB-TCNQF 2

in the case of the other TMB CT co-crystals, TMB and the acceptor molecule are aligned along their long axes. In the present case this implies that there is disorder in the reciprocal arrangement of the molecules, as $\mathrm{TCNQF}_{2}$ has two equivalent positions, rotated by $180^{\circ}$ around the axis perpendicular to the molecular plane.

The analysis of the vibrational spectra indicate that TMB-TCNQF 2 is a quasi-ionic CT crystal, and that the stack is indeed dimerized. The top of Fig. 6 shows the IR spectra polarized perpendicularly to the stack axis, where the charge-sensitive in-plane modes are observed. We shall not use the TCNQ CN antisymmetric stretching vibration (right top panel of the Fig. 6) to estimate $\rho$ since it is well know that it gives unreliable (generally overestimated) value. In fact, $\mathrm{CN}$ antisymmetric stretching suffers from uncertainty about the correct assignment and is subject to extrinsic effects due to the interactions with the surrounding molecules $[16,36]$. We therefore use the $\mathrm{TCNQF}_{2}$ ring stretching vibrations, as singled out and calibrated in Ref. [15]. The frequencies of the two safely identified charge sensitive modes are reported directly in Fig.6. The resulting $\rho$ is 0.66 . The bottom panel of Fig. 6 compares the TMB-TCNQF 2 IR spectra polarized along the stack with the Raman spectrum. It is seen that the IR spectrum is dominated by strong absorptions at the same frequencies as the Raman bands. These absorptions are induced by the e-mv mechanism, and are a clear signature of the stack dimerization [32].

The crystal structure of TMB-TCNQF 4 is somewhat different from that of TMB-TCNQ and TMB-TCNQF 2 : As shown in Table II and Fig. 7, it crystallizes in the monoclinic system $C 2 / m\left(C_{2 h}^{3}\right)$, with two DA pairs per unit cell. The stack axis is $c$, and the mean planes through the TMB and $\mathrm{TCNQF}_{4}$ molecules are inclined by $85.54(2)^{\circ}$ and $86.65(2)^{\circ}$, respectively, with respect to stack axis. The molecules reside on inversion center, so that the stack appears to be regular.

The IR spectra polarized perpendicular to the stack (Fig. 8, top panel) indicates that, as expected, TMB$\mathrm{TCNQF}_{4}$ is more ionic than TMB-TCNQ and TMB$\mathrm{TCNQF}_{2}$. We have followed the procedure already described for TMB-TCNQF 2 : Three charge sensitive bands have been identified in the CC stretching region [15], and

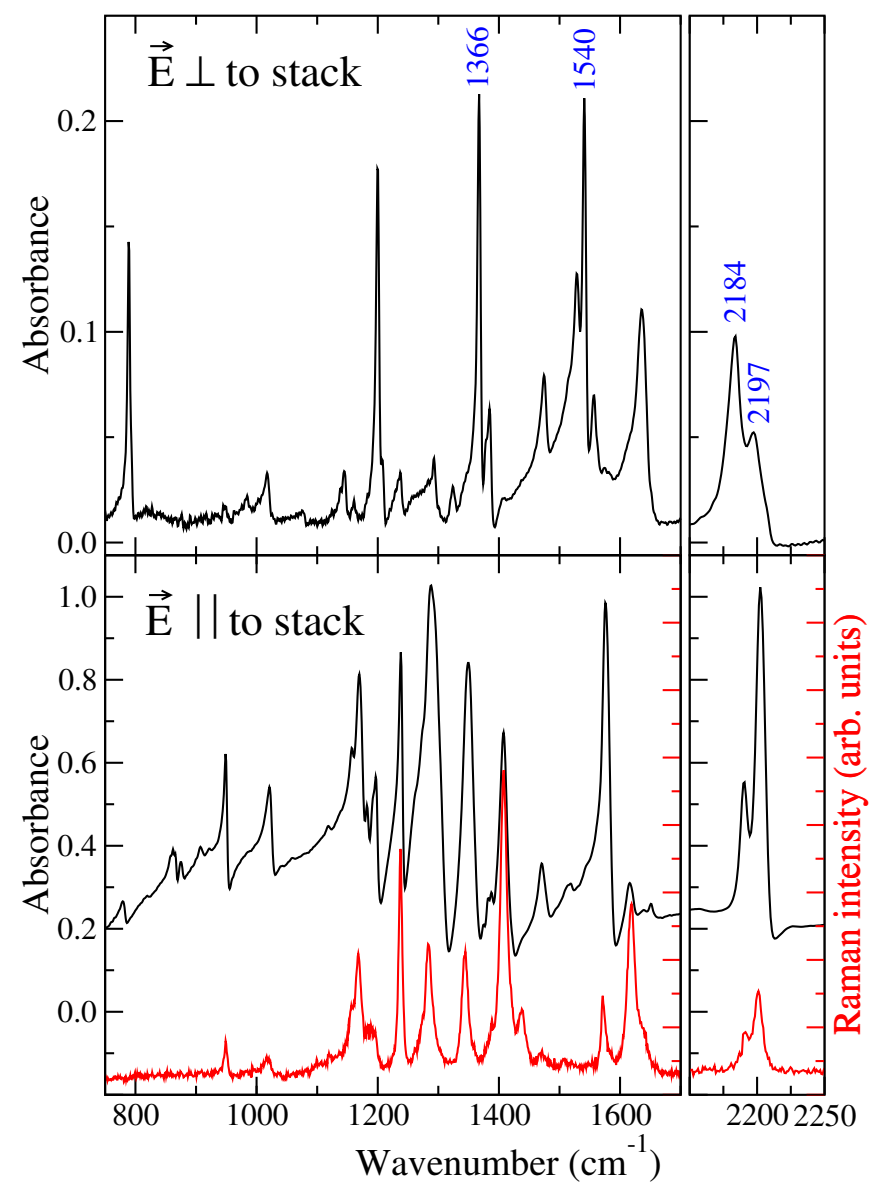

FIG. 6. Polarized IR and Raman spectra of TMB-TCNQF 2

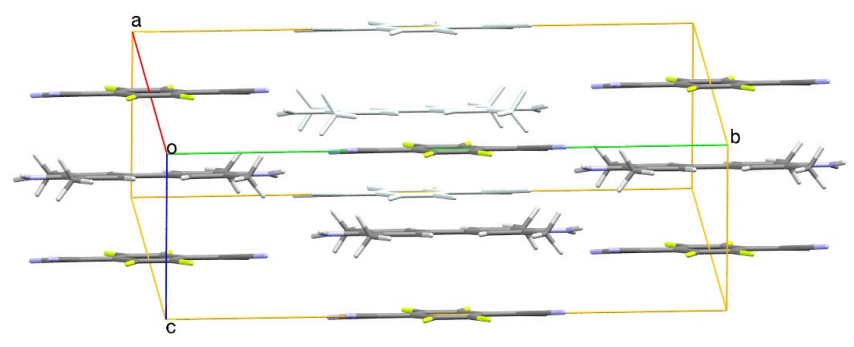

FIG. 7. Crystal structure of TMB-TCNQF 4

their frequencies (reported in the left top panel of the Figure) indicate a ionicity of 0.91 . On the other hand, the IR spectra polarized parallel to the stack (bottom panel of Fig. 8) provide a somewhat unexpected result: The presence of strong IR absorptions at the same frequencies of the main Raman band would indicate that the stack is dimerized, like that of $\mathrm{TMB}-\mathrm{TCNQF}_{2}$, but this is in contrast with the X-ray crystal structure. X-ray probe long-range order, whereas vibrational spectroscopy probe the local (DA pair) structure. One might be thinking of a disordered structure, with opposite distortion, so that on average the stack appears as regular. But this seems to be incompatible with the X-ray atomic thermal ellip- 


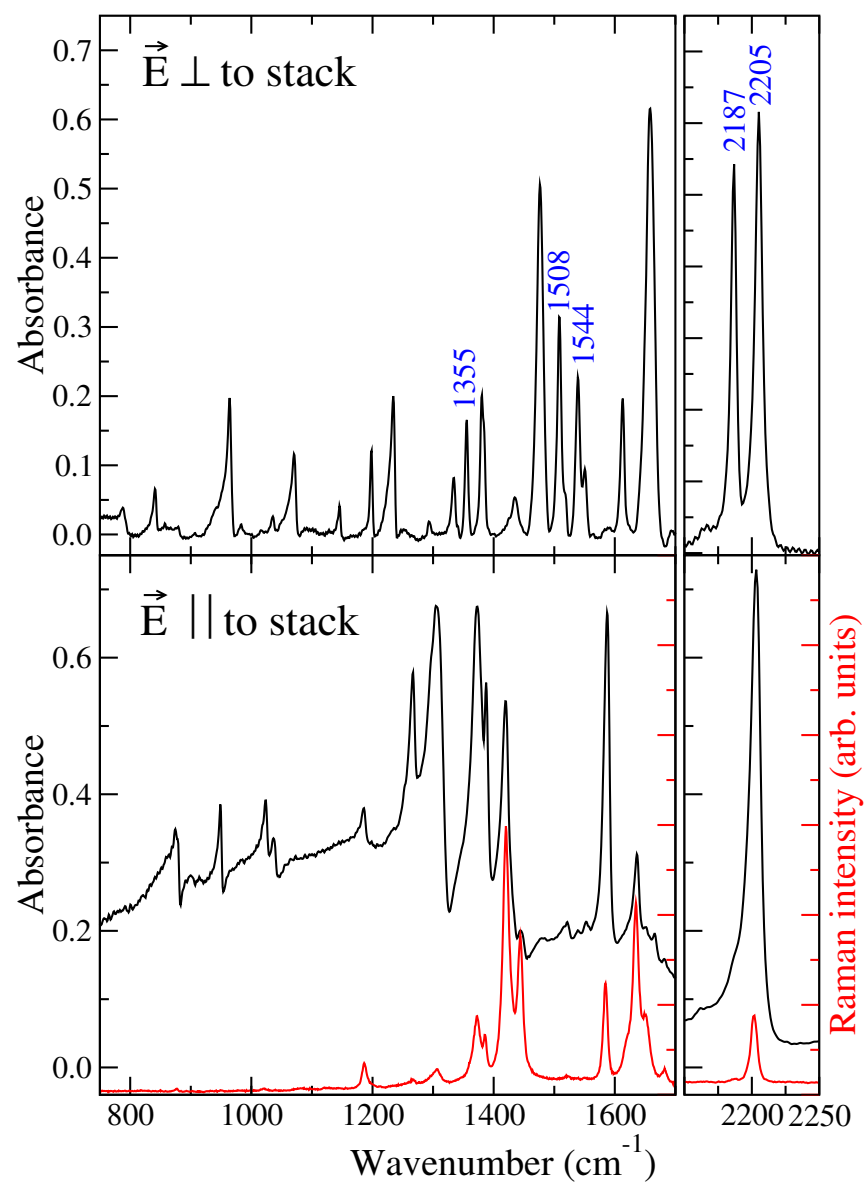

FIG. 8. Polarized IR and Raman spectra of TMB-TCNQF 4

soids. Some of us have already encountered a similar case [37], but we believe that the problem needs to be further investigated before drawing definite conclusions.

\section{Charge transfer transition}

We finally investigate the near-IR spectral region, where the $\mathrm{CT}$ transition occurs. In this case one has to resort to reflectance spectra, as the absorbance saturates in correspondence to the transition. Due to the strong optical anisotropy of the crystals, only reflectance spectra polarized parallel to the stack axis afforded sufficiently high reflectance. The reflectance spectra of all CT crystals studied here are reported in Fig. 9, together with the spectrum of TMB-TCNQ. We remark that the latter is considerably different from the one reported in Ref. [30], as the peak frequency is shifted by more than $2500 \mathrm{~cm}^{-1}$. Careful and repeated control allowed us to conclude that the present one is the correct spectrum, reproducible on different samples, whereas we never obtained again the spectrum reported in Ref. [30]. We are unable to explain the origin of the error, if is due to picking up a different polymorph [38], or if we incurred in some instrumental artifact, as in the present measure-

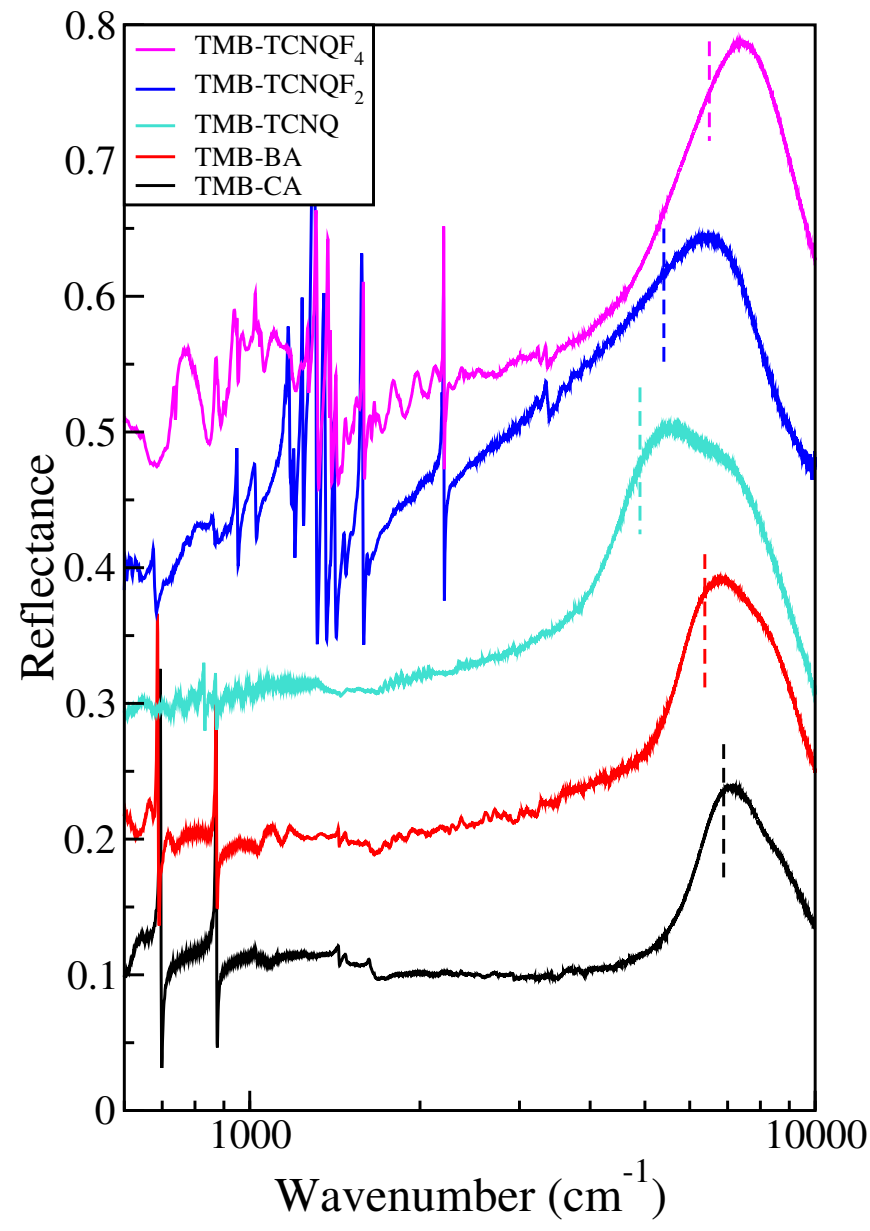

FIG. 9. Reflectance spectra of TMB-Acceptor CT crystals, with polarization parallel to the stack axis and extension to the near-IR region $\left(10000 \mathrm{~cm}^{-1}\right)$. From bottom to top the spectra refer to: TMB-CA, TMB-BA, TMB-TCNQ, TMB$\mathrm{TCNQF}_{2}, \mathrm{TMB}_{\mathrm{TCNQF}}$. For the sake of clarity, each spectum is offset by about 0.1 in the reflectance scale. Notice the logarithmic wavenumber scale. The dashed lines indicate the oscillator frequency of the Drude-Lorentz model.

ments we use a slightly different setup. As explained in Section II C, the reflectance values cannot be considered as absolute.

In Fig. 9 we also report the mid-IR region, down to $600 \mathrm{~cm}^{-1}$, where one can detect the out-of-plane vibrational modes and, in the case of TMB-TCNQF 2 and TMB-TCNQF 4 , the e-mv induced modes previously discussed. Due to current experimental limitations, the high frequency limit is $10000 \mathrm{~cm}^{-1}$, preventing a reliable Kramers-Kronig transformation of the data. We have therefore directly fitted the reflectance spectra with a Drude-Lorentz model, using the program RefFIT [39]. In the case of TMB-CA, BA, and TCNQ the CT band appears to have some structure, probably of vibronic origin [40]. We tried to fit the data with a single or two Lorentians, but the frequency of the main peak does not change significantly. And indeed the only datum that 
we use from the fitting is the position of the oscillator transverse frequency $\omega_{\mathrm{CT}}$. The obtained values are summarized in Table III, and visually marked by a dashed vertical line in Fig. 9.

According to exact model calculations for mixed regular stack CT crystals [41], $\omega_{\mathrm{CT}} /(\sqrt{2} t)$ is an U-shaped universal function of $\rho$, with a minimum around 0.6, where the optical gap closes and the system becomes intrinsically unstable. A glance at Fig. 9 shows that $\omega_{\mathrm{CT}}(\rho)$ indeed follows this trend, meaning that $t$, the DA CT integral, changes little along the series.

TABLE III. Basic experiment-derived parameters of the TMB-acceptor series.

\begin{tabular}{l|ccccc}
\hline \hline System & $d(\AA)$ & $V_{\text {cell }}\left(\AA^{3}\right)$ & $\rho$ & $\omega_{\mathrm{CT}}(\mathrm{eV})$ & $t(\mathrm{eV})$ \\
\hline TMB-CA & 3.322 & 1083.37 & 0.14 & 0.85 & 0.19 \\
TMB-BA & 3.254 & 1133.96 & 0.16 & 0.79 & 0.21 \\
TMB-TCNQ & 3.354 & 1161.33 & 0.29 & 0.60 & 0.29 \\
TMB-TCNQF $_{2}$ & $3.378 / 3.160$ & 1181.61 & 0.66 & 0.67 & - \\
TMB-TCNQF $_{4}$ & 3.340 & 1206.26 & 0.91 & 0.81 & 0.17 \\
\hline \hline
\end{tabular}

In Table III we compare the experimentally derived basic parameters of the TMB-Acceptor serie, namely $d$, the interplanar distance between D and A along the stack, $\rho, \omega_{\mathrm{CT}}$, and $t$, the latter obtained from the universal curve of Ref. [41] and the $\rho$ value estimated by IR spectroscopy. The $t$ value is missing in the case of TMB$\mathrm{TCNQF}_{2}$, because the stack is dimerized, but also because the calculations suffer from a high degree of uncertainty between $\rho \simeq 0.4$ and $\rho \simeq 0.7$. The interplanar distance is around $3.3 \AA$ for all the systems, by considering the average in the case of the dimerized stack of TMB-TCNQF 2 . The dimerization amplitude of the latter, $\delta=\left(d_{2}-d_{1}\right) /\left(d_{2}+d_{1}\right)=0.033$ is somewhat larger than that of the low-temperature phase of TTF$\mathrm{CA}, \delta=0.025$ [35]. The ionicity, on the other hand, span from the quasi-neutral TMB-CA to quasi-ionic TMB$\mathrm{TCNQF}_{4}$, whereas $t$ remains between about 0.2 and 0.3 $\mathrm{eV}$.

\section{DISCUSSION AND CONCLUSIONS}

The results of the previous Section have shown that the combination of TMB donor with a series of five acceptors of increasing electron affinity provides a extensive exploration of the phase space of ms-CT crystals. At room temperature we have three systems on the neutral side, TMB-CA, TMB-BA and TMB-TCNQ, with a regular stack. Around $200 \mathrm{~K}$ the third system undergoes a valence instability, with dimerization of the stack, but remains on the neutral side $(\rho \simeq 0.4)$ [30]. TMB$\mathrm{TCNQF}_{2}$ also has intermediate ionicity $(\rho=0.66)$, but is on the ionic side, and the stack is dimerized. Finally $\mathrm{TMB}^{-T C N Q F}{ }_{4}$ is nearly ionic $(\rho=0.91)$, and the stack is presumably regular with some degree of local disorder (dimerization). Preliminary investigations have shown that, apart from TMB-TCNQ, no other crystal undergoes phase transitions down to $77 \mathrm{~K}$.

As already mentioned in the Introduction, the phase space of ms-CT crystals has been rationalized many years ago on the basis of a one-dimensional (1D) extended Hubbard model, including intra- and inter-molecular electron-phonon coupling [42]. Briefly, we can have N and I ground states, but on the I side the stack is intrinsically unstable towards dimerization due to the Peierls mechanism. Since the effect of the Peierls coupling is maximum for systems of intermediate ionicity, we can observe neutral $(\rho \gtrsim 0.3)$ crystals with dimerized stack, and fully ionic $(\rho \simeq 1)$ crystals with regular stacks. In systems made up by similar D and A molecules, like in the present series, we can assume the electron-phonon coupling as roughly constant, so that the position of a given system within the phase space can be understood in terms of the electronic parameters only. Within the extended Hubbard model such parameters are: $z=\left[\left(I_{D}-A_{A}\right)+V\right] / 2$, half the energy required to form a ionic pair $\left(I_{D}\right.$ is the $\mathrm{D}$ ionization potential, $A_{A}$ the $\mathrm{A}$ electron affinity, and $V$ is the nearest-neighbor D-A Coulomb interaction); $t$, the DA hopping or CT integral; and $M$, the $3 \mathrm{D}$ crystal Madelung energy that is introduced into the 1D Hamiltonian at mean-field level.

Some of us have recently shown that the parameters entering the extended Hubbard model can be evaluated by simple DFT calculations on isolated DA pairs, while DFT calculation on D/A molecules and their ions constitute the basis for the computation of the 3D Madelung energy [24]. We have then undertaken such calculations for the five systems at hand, aimed at extracting the $z$, $t, V$ and $M$ parameters, and provide a rationale for the found experimental behavior. The results are collected in Table IV, where the difference $\epsilon_{c}=V-2 M$ is also reported for convenience.

TABLE IV. Basic computed parameters of the TMB-acceptor series. Parameter values in $\mathrm{eV}$.

\begin{tabular}{|c|c|c|c|c|c|}
\hline System & $z$ & $\bar{t}$ & $\bar{V}$ & $M$ & $\epsilon_{c}$ \\
\hline TMB-CA & 0.49 & 0.26 & -2.27 & -1.49 & 0.70 \\
\hline TMB-BA & 0.47 & 0.31 & -2.34 & -1.55 & 0.75 \\
\hline TMB-TCNQ & 0.28 & 0.23 & -2.03 & -1.33 & 0.62 \\
\hline $\mathrm{TMB}^{\mathrm{TCNQF}}{ }_{2}$ & 0.16 & 0.27 & -1.91 & -1.25 & 0.59 \\
\hline $\mathrm{TMB} \mathrm{TCNQF}_{4}$ & 0.02 & 0.07 & -1.89 & -0.96 & 0.03 \\
\hline
\end{tabular}

The monotone decrease of the $z$ values in the first column of Table IV essentially reflects the difference in the electron affinity of the acceptors, slightly affected by an equally monotone decrease of $V$ (third column). The computed $t$ values (second column) are in satisfactory agreement with the experimentally derived ones in the fifth column of Table III, also considering the uncertainties in both estimates. In particular, the uncertainty in the calculation is larger for ionic system, as the $t$ estimate is based on the computed values of the ionicity and of the singlet-triplet gap of an isolated DA pair [24]. The gap is small for ionic system (e.g., $0.12 \mathrm{eV}$ for TMB-TCNQF 4 ), 
so that errors in the calculation of the (large) singlet and triplet energies spoil the small difference. We can conclude that the $t$ values are in any case comprised between 0.2 and $0.3 \mathrm{eV}$, with a slight decrease for TMB-TCNQF 4 , probably due to the inclination of the molecules with respect to the stack axis.

The Madelung energy in the fifth column of Table IV varies from -1.49 to -0.96 , essentially reflecting the $3 \mathrm{D}$ packing of the different structures. We notice that the Madelung energy is smallest for the most ionic compound, following the corresponding volume increase (cf. Table III), again a likely consequence of the inclination of the molecules with respect to stack axis. These differences in $t$ and in $M$ are in any case not sufficient to affect the increase in $\rho$ with the decrease of $z$, as it happens instead in the BTBT-TCNQF ${ }_{\mathrm{x}}$ series [43].

The $\epsilon_{c}$ values (fourth column of Table IV) are also instructive. Both $V$ and $M$ correspond to attractive interactions, and as such have negative values. Their difference reflects the inter-stack interactions, and positive $\epsilon_{c}$ point to the presence of dominant attractive inter-stack interactions, larger than the intra-stack one. This is the case for all the TMB series, but in other cases negative $\epsilon_{c}$ have been found $[24,43]$, typically corresponding to crystals with one DA pair per unit cell. These crystals may exhibit dominant D-D and/or A-A interstack repulsions along directions perpendicular to the stack. Based on the extensive number of investigated systems, we advance the plausible hypothesis that largely positive $\epsilon_{c}$ values imply a lattice contraction upon decreasing temperature, whereas strongly negative values point to a lattice instability [43].

One perspective application of ms-CT crystals is their use as semiconductors with balanced electron and hole mobility, as first suggested on the basis of DFT calculations and band theory [44]. A quasi neutral $(\rho \approx 0.3)$ ms-CT crystal, DBTTF-TCNQ, does indeed exhibit appreciable hole and electron mobility $[45,46]$, but other CT crystals do not. As a matter of fact, in ms-CT crystals electron correlations are important [42], and band theory might be inadequate to account for their behavior. From this point of view, the present series of ms-CT crystals offer a very good opportunity to test theoretical models, given the differences of the encompassed parameter values in an otherwise similar packing arrangement. Mobility measurements are currently under way in our laboratories.

We finally address the issue of potential ferroelectricity in the TMB series. Indeed, ms-CT crystals may exhibit ferroelectricity, that can occur by the usual mechanism of ionic displacement, or can be due to a new polarization mechanism of the electronic cloud, the so-called "electronic" ferroelectricity [13]. In order to be potentially ferroelectric ms-CT crystals must lack the inversion center, that is, the stack has to be dimerized, and in-phase dimerization has to occur in case of two stacks per unit cell. TTF-CA is the prototype of electronic ferroelectricity, but only below $80 \mathrm{~K}$ [5], when NIT occurs and the stack dimerizes. Ferroelectricity at room temperature has been claimed for other ms-CT crystals [47], but the results have been recently questioned, since the crystal structures are centro-symmetric and there is no local stack dimerization [48].

According to X-ray, TMB-TCNQF 4 stack is not dimerized, but vibrational spectroscopy suggests the opposite. If the discrepancy is due to a disordered local dimerization, then an electric field might induce ferroelecticity. On the other hand, TMB-TCNQF 2 stack is certainly dimerized at room temperature, and has a degree of CT around 0.6, like TTF-CA at low temperature [5]. Unfortunately, the arrangements of the two DA pairs in the unit cell is anti-ferroelectric (Fig. 5). We notice that the structural class of TMB-TCNQF ${ }_{2},\left(P 2_{1}, Z=2(1)\right)$, is the same as that calculated for the low-temperature phase of TMB-TCNQ [30]. The structure was only calculated, because the crystal is damaged at the transition, and at the time of Ref. [30] writing it was not possible to solve the low-temperature X-ray structure. Now such structure has been obtained [49], and is different from the calculated one, with a ferroelectric arrangement of the DA pairs in the unit cell. Since the crystal is in any case damaged at the transition, the potential ferrolectricity is likely spoiled. We then suggest to investigate the possibility of photoinduced ferroelectricity, as discovered for TTF-CA [50], with the advantage of operating at room temperature or slightly below.

To summarize, we believe that the TMB-Acceptor series studied in this paper presents several appealing perspectives, and has all the prerequisites to become an ideal investigative playground, much like the TTFhaloquinone series has been [8].

\section{ACKNOWLEDGMENTS}

Support to the reserach by Parma University is acknowledged. The computational part has been supported by CINECA Consortium through Grant No. IsC50-HP10C8YPOY. C.R. acknowledges support from MINECO of the Spanish Government (CTQ2016- 80030$\mathrm{R}$ and SEV-2015-0496). A.G. thanks G. D'Avino for providing the MESCAL program and for enlightening discussions, and A. Painelli for her continuous support.
[1] G. Saito and Y. Yoshida, Bull. Chem. Soc. Jpn. 80, 1 (2007).
[2] S. Tomic and M.Dressel, Rep. Prog. Phys. 78, 096501 (2015). 
[3] R. K. S. Horiuchi, Y. Okimoto and Y. Tokura, Science 229, 299 (2003).

[4] F. Kagawa, S. Horiuchi, M. Tokunaga, J. Fujioka, and Y. Tokura, Nature Phys. 6, 169 (2010).

[5] K. Kobayashi, S. Horiuchi, R. Kumai, F. Kagawa, Y. Murakami, and Y. Tokura, Phys. Rev. Lett. 108, 237601 (2012).

[6] J. B. Torrance, J. E. Vazquez, J. J. Mayerle, and V. Y. Lee, Phys. Rev. Lett. 46, 253 (1981).

[7] J. B. Torrance, A. Girlando, J. J. Mayerle, J. I. Crowley, V. Y. Lee, P. Batail, and S. J. LaPlaca, Phys. Rev. Lett. 47, 1747 (1981).

[8] M. Masino, N. Castagnetti, and A. Girlando, Crystals 7, 108 (2017).

[9] E. Dagotto, Science 309, 257 (2005).

[10] J. Tsutsumi, H. Matsui, T. Yamada, R. Kumai, and T. Hasegawa, J. Phys. Chem. C 116, 23957 (2012).

[11] J. Zhang, W. Xu, P. Sheng, G. Zhao, and D. Zhu, Acc. Chem. Res. 50, 1654 (2017).

[12] T. Morimoto, T. Miyamoto, and H. Okamoto, Crystals 7, 132 (2017).

[13] S. Horiuchi, K. Kobayashi, R. Kumai, and S. Ishibashi, Chem. Lett. 43, 26 (2014).

[14] P. Hu, K. Du, F. Wei, H. Jiang, and C. Kloc, Cryst. Growth Des. 16, 3019 (2016).

[15] T. Salzillo, M. Masino, G. Kociok-Köhn, D. Di Nuzzo, E. Venuti, R. G. Della Valle, D. Vanossi, C. Fontanesi, A. Girlando, A. Brillante, and E. Da Como, Cryst. Growth Des. 16, 3028 (2016).

[16] A. Morherr, S. Witt, A. Chernenkaya, J.-P. Bcker, G. Schnhense, M. Bolte, and C. Krellner, Physica B: Cond. Matter 496, 98 (2016).

[17] G. D'Avino, A. Painelli, and Z. G. Soos, Crystals 7, 144 (2017).

[18] R. Laudise, C. Kloc, P. Simpkins, and T. Siegrist, J. Cryst. Growth 187, 449 (1998).

[19] APEX2, SAINT, and SADABS (Bruker AXS Inc., Madison, Wisconsin, U.S.A.).

[20] G. M. Sheldrick, Acta Cryst. A 71, 3 (2015).

[21] G. M. Sheldrick, Acta Cryst. C 71, 3 (2015).

[22] C. F. Macrae, I. J. Bruno, J. A. Chisholm, P. R. Edgington, P. McCabe, E. Pidcock, L. Rodriguez-Monge, R. Taylor, J. van de Streek, and P. A. Wood, J. Applied Cryst. 41, 466 (2008).

[23] "Jmol: An open-source Java viewer for chemical structures in 3D," http://www.jmol.org (2017).

[24] F. Delchiaro, A. Girlando, A. Painelli, A. Bandyopadhyay, S. K. Pati, and G. D'Avino, Phys. Rev. B 95, 155125 (2017).

[25] M. J. Frisch, G. W. Trucks, H. B. Schlegel, G. E. Scuseria, M. A. Robb, J. R. Cheeseman, G. Scalmani, V. Barone, B. Mennucci, G. A. Petersson, H. Nakatsuji, M. Caricato, X. Li, H. P. Hratchian, A. F. Izmaylov, J. Bloino, G. Zheng, J. L. Sonnenberg, M. Hada, M. Ehara, K. Toyota, R. Fukuda, J. Hasegawa, M. Ishida, T. Nakajima, Y. Honda, O. Kitao, H. Nakai, T. Vreven, J. A. Montgomery, Jr., J. E. Peralta, F. Ogliaro, M. Bearpark, J. J. Heyd, E. Brothers, K. N. Kudin, V. N. Staroverov, R. Kobayashi, J. Normand, K. Raghavachari, A. Rendell, J. C. Burant, S. S. Iyengar, J. Tomasi, M. Cossi, N. Rega, J. M. Millam, M. Klene, J. E. Knox, J. B. Cross, V. Bakken, C. Adamo, J. Jaramillo, R. Gomperts, R. E. Stratmann, O. Yazyev, A. J. Austin, R. Cammi, C. Pomelli, J. W. Ochterski, R. L. Martin, K. Morokuma,
V. G. Zakrzewski, G. A. Voth, P. Salvador, J. J. Dannenberg, S. Dapprich, A. D. Daniels, . Farkas, J. B. Foresman, J. V. Ortiz, J. Cioslowski, and D. J. Fox, "Gaussian09 Revision E.01," (2009), Gaussian Inc. Wallingford CT 2009.

[26] G. D'Avino, L. Muccioli, C. Zannoni, D. Beljonne, and Z. G. Soos, J. Chem. Theor. Comput. 10, 4959 (2014).

[27] V. K. Belsky, O. N. Zorkaya, and P. M. Zorky, Acta Cryst. A 51, 473 (1995).

[28] A. Girlando and C. Pecile, J.Chem. Soc. Faraday II 71 (1975).

[29] A. Girlando, I. Zanon, R. Bozio, and C. Pecile, J.Chem.Phys. 68 (1978).

[30] N. Castagnetti, G. Kociok-Köhn, E. Da Como, and A. Girlando, Phys. Rev. B 95, 024101 (2017).

[31] P. Ranzieri, M. Masino, and A. Girlando, J. Phys. Chem. B 111, 12844 (2007).

[32] C. Pecile, A. Painelli, and A. Girlando, Mol. Cryst. Liq. Cryst. 171, 69 (1989).

[33] M. Masino, A. Girlando, and Z. Soos, Chem. Phys. Lett. 369, 428 (2003).

[34] A. Girlando, M. Masino, A. Painelli, N. Drichko, M. Dressel, A. Brillante, R. G. Della Valle, and E. Venuti, Phys. Rev. 78, 045103 (2008).

[35] M. LeCointe, M. H. Lemeé-Cailleau, H. Cailleau, B. Toudic, L. Toupet, G. Heger, F. Moussa, P. Schweiss, K. H. Kraft, and N. Karl, Phys. Rev. B 51, 3374 (1995).

[36] M. Meneghetti and C. Pecile, J. Chem. Phys. 84, 4149 (1986).

[37] S. A. Bewick, R. A. Pascal, D. M. Ho, Z. G. Soos, M. Masino, and A. Girlando, J. Chem. Phys. 122, 024710 (2005).

[38] Y. Iwasa, T. Koda, S. Koshlhara, T. Tokura, and G. Saito, Mol. Cryst. Liq. Cryst. 216, 195 (1992).

[39] A. Kuzmenko, "Reffit," https://sites.google.com/ site/reffitprogram/ (2015).

[40] K. Yakushi and H. Kuroda, Chem. Phys. Lett. 111, 165 (1984).

[41] A. Painelli and A. Girlando, J. Chem. Phys. 87, 1705 (1987).

[42] A. Painelli and A. Girlando, Phys. Rev. B 37, 5748 (1988).

[43] N. Castagnetti, A. Girlando, M. Masino, C. Rizzoli, and C. Rovira, Cryst. Growth Des. 17, 6255 (2017).

[44] L. Zhu, Y. Yi, Y. Li, E.-G. Kim, V. Coropceanu, and J.-L. Brédas, J. Amer. Chem. Soc. 134, 2340 (2012).

[45] K. P. Goetz, J. Tsutsumi, S. Pookpanratana, J. Chen, N. S. Corbin, R. K. Behera, V. Coropceanu, C. A. Richter, C. A. Hacker, T. Hasegawa, and O. D. Jurchescu, Adv. Electron. Mater. 2, 1600203 (2016).

[46] A. Girlando, Adv. Electron. Mater. 3, 1600437 (2017).

[47] A. S. Tayi, A. K. Shveyd, A. C. H. Sue, J. M. Szarko, B. S. Rolczynski, D. Cao, T. J. Kennedy, A. A. Sarjeant, C. L. Stern, W. F. Paxton, W. Wu, S. K. Dey, A. C. Fahrenbach, J. R. Guest, H. Mohseni, L. X. Chen, K. L. Wang, J. F. Stoddart, and S. I. Stupp, Nature 488, 485 (2012).

[48] G. D'Avino, M. Souto, M. Masino, J. K. H. Fischer, I. Ratera, X. Fontrodona, G. Giovannetti, M. J. Verstraete, A. Painelli, P. Lunkenheimer, J. Veciana, and A. Girlando, Nature 547, E9 (2017), brief Communication Arising.

[49] N. Castagnetti and F. Mezzadri, (private communication). 
[50] T. Morimoto, T. Miyamoto, and H. Okamoto, Crystals 7 (2017). 